\title{
NON-SIMPLICITY OF LOCALLY FINITE BARELY TRANSITIVE GROUPS
}

\author{
by B. HARTLEY* and M. KUZUCUOĞLU
}

(Received 11th September 1995)

\begin{abstract}
We answer the following questions negatively: Does there exist a simple locally finite barely transitive group (LFBT-group)? More precisely we have: There exists no simple LFBT -group. We also deal with the question, whether there exists a LFBT-group $G$ acting on an infinite set $\Omega$ so that $G$ is a group of finitary permutations on $\Omega$. Along this direction we prove: If there exists a finitary LFBT-group $G$, then $G$ is a minimal non-FC p-group. Moreover we prove that: If a stabilizer of a point in a LFBT-group $G$ is abelian, then $G$ is metabelian. Furthermore $G$ is a $p$-group for some prime $p, G / G^{\prime} \cong C_{p \infty}$, and $G^{\prime}$ is an abelian group of finite exponent.
\end{abstract}

1991 Mathematics subject classification: 20F50, 20B07, 20E22, $20 \mathrm{E} 32$.

Let $\Omega$ be an infinite set. Then a transitive subgroup $G$ of $\operatorname{Sym}(\Omega)$ is said to be barely transitive if every orbit of every proper subgroup of $G$ is finite. More generally, we say that a group $G$ is barely transitive if it can be represented as a barely transitive subgroup of $\operatorname{Sym}(\Omega)$ for some infinite set $\Omega$. This is easily seen to be equivalent to the condition that $G$ has a subgroup $H$ of infinite index such that $\bigcap_{g \in G} H^{g}=\{1\}$ and such that $|K: K \cap H|$ is finite for every proper subgroup $K$ of $G$. Throughout this article, if $G$ is a barely transitive group, then $H$ will denote a fixed subgroup of $G$ with the above properties.

In this article, we shall study locally finite barely transitive groups, which we shall call LFBT-groups. Metabelian LFBT-groups were constructed by B. Hartley in [4] and [5]. It is unknown whether perfect LFBT-groups exists. We shall prove that there are no simple LFBT-groups; and, as a consequence, improve on some of the results in [8].

Theorem 1. There exists no simple LFBT-group.

It is also natural to ask whether there exists a LFBT-group $G$ acting on an infinite set $\Omega$ so that $G$ is a group of finitary permutations on $\Omega$.

Theorem 2. If there exists a finitary LFBT-group $G$, then $G$ is a minimal non-FC, p-group.

- The Society is saddened by the death of Professor Brian Hartley. 
Theorem 3. If $G$ is a finitary permutation group on $\Delta$ and $G=\left\langle g_{i}\right| g_{i}^{p}=1$, $i=1,2,3 \ldots)$, then $G$ is not a LFBT-group on $\Delta$.

In [7], it was asked how restrictions on $H$ affect the structure of a LFBT-group. We shall prove the following result.

Proposition 1. Let $G$ be a LFBT-group. If $H$ is abelian, then $G$ is metabelian. Furthermore

(i) $G$ is a p-group, p prime.

(ii) $G / G^{\prime}$ is isomorphic to $C_{p \infty}$.

(iii) $G^{\prime}$ is an abelian group of finite exponent.

It should be pointed out that in each of the LFBT-groups constructed in [4] and [5], the subgroup $H$ is abelian.

The second author would like to thank Prof. S. Thomas for some valuable discussions. He would also like to thank Prof. J. Roseblade for his major improvements in the exposition of this paper.

\section{Proofs of the results}

We will begin by collecting together some of the basic properties of LFBT-groups. Complete proofs of these results can be found in [8].

$$
G \text { has no proper subgroup of finite index. }
$$

Suppose

$$
H=H_{0}<H_{1}<H_{2}<\ldots<H_{n} \ldots
$$

is a chain of subgroups of $G$ above $H$. Since $\left|H_{n}: H\right|$ is finite, there is a finite subgroup $L_{n}$ of $H_{n}$ with $H_{n}=H L_{n}$. Let $F_{n}=\left\langle L_{1}, \ldots, L_{n}\right\rangle$.

We have

$$
F_{1}<F_{2}<\ldots<F_{n}<\ldots
$$

and

$$
\begin{aligned}
& \qquad H_{n}=H F_{n} \\
& \text { Evidently } G=\bigcup_{n} H_{n}
\end{aligned}
$$


follows from the fact that $K \leq G$ and $|K: K \cap H|=\infty$ implies $K=G$. Again by the same reason we have

$$
G=\bigcup_{n} F_{n}
$$

$$
\text { Further if } X<G \text {, then } X \leq H_{n} \text { for some } n
$$

Now suppose that there is no simple LFBT-group. Since it is clear that any simple homomorphic image of $G$ would have to be a LFBT-group, it follows that $G$ has no maximal normal subgroup. Hence $G$ is a union of proper normal subgroups. In particular

$$
F_{n}^{G}<G
$$

Proposition 2. Let $G$ be a LFBT-group. Then either $G$ is a p-group for some prime $p$ or there are infinitely many primes dividing the order of the elements of $G$.

Proof. By (3) and (6) we have $F_{1} \leq F_{2} \leq \ldots$ a sequence of finite subgroups of $G$ such that $G=\cup_{i=1}^{\infty} F_{i}$. Assume that $G$ is not a $p$-group and there are only finitely many primes, say $p_{1}, \ldots, p_{k}$, dividing the order of the elements of $G$. Let $S_{i 1}$ be a Sylow $p_{i}$-subgroup of $F_{1}$ and let $S_{i 2}$ be a Sylow $p_{i}$-subgroup of $F_{2}$ containing $S_{i 1}$, etc. Then $S_{i}=\cup_{j=1}^{\infty} S_{i j}$ is a maximal $p_{i}$-subgroup of $G$. We shall show that $G=\left\langle S_{1}, \ldots, S_{k}\right\rangle$. The group $F_{j} \cap\left\langle S_{1}, \ldots, S_{k}\right\rangle$ contains the groups $S_{1 j}, \ldots, S_{k j}$, hence equals to $F_{j}$, for all $j$. This implies that $G$ is generated by a finite number of proper maximal $p_{i}$-subgroups which is impossible by [8, Lemma 2.10]. Thus infinitely many primes must divide the order of the elements of $G$.

Proof of Theorem 1. Assume that there exists a simple LFBT-group $G$. By (6) $G$ is countable and by [8, Lemma 2.10], $G$ cannot be generated by two proper subgroups. Then by [1, Corollary 1.9] such a group can be embedded in a finitary linear group $F G L(V)$ on a vector space $V$ over a field of characteristic $p$.

By [2, Theorem B], for an infinite simple periodic group $G$ of finitary transformations on a space over a field of characteristic $p$ the following are valid:

(1) If $p=0$, then for each finite subgroup $K$ of $G$, there exists a finite quasisimple subgroup $H$ that contains $K$ and is such that $K \cap Z(H)=\{1\}$.

(2) If $p>0$, then for each finite subgroup $K$ of $G$, there exists a finite subgroup $H$ that contains $K$ and is such that $H=H^{\prime}, H / O_{p}(H)$ is a quasisimple group and $K \cap S(H)=\{1\}$ where $S(H)$ is the maximal soluble normal subgroup of $H$.

In the first case, $G$ has a sequence of finite subgroups $G_{1}<G_{2}<\ldots$ where $G=\cup_{i=1}^{\infty} G_{i}$ and $G_{i} \cap Z\left(G_{i+1}\right)=\{1\}$ (i.e. A Kegel sequence $\left.\left(G_{i}, Z\left(G_{i}\right)\right) i=1,2,3, \ldots\right)$. By 
[8, Lemma 4.2], $G$ cannot be a barely transitive group. (For details about Kegel sequences and reductions on Kegel sequences, see [6].)

For the second case, let $G=\cup_{i=1}^{\infty} G_{i}$, where $G_{i} / O_{p}\left(G_{i}\right)$ are finite quasisimple groups. We shall show that there exists an element $x$ in $G$ such that $C_{G}(x)$ involves an infinite non-linear locally finite simple group; then we shall get a contradiction. Let $\bar{G}_{i}=G_{i} / O_{p}\left(G_{i}\right)$.

By using the classification of finite simple groups and reduction on Kegel sequences we may assume that

(i) each $\bar{G}_{i} / Z\left(\bar{G}_{i}\right)$ is an alternating group or

(ii) each $\bar{G}_{i} / Z\left(\bar{G}_{i}\right)$ is a classical group of fixed Lie type over a field of characteristic $p_{i}$.

For (i), the centralizer $C_{G}(x)$ of any element $x$ of order prime to $p$ involves an infinite non-linear locally finite simple group. See [6, Lemma 2.5].

For (ii), let $\left\{p_{i}: i \in N\right\}$ be the set of primes that appear as characteristic of the fields. If one of the primes, say $p_{j}$, in this set appears infinitely often, then we choose an element of prime order relatively prime to $p$ and $p_{j}$. Existence of this element is guaranteed by Proposition 2.

If none of the primes appears as a characteristic of the fields infinitely many times, then we may assume that each prime appears as a characteristic only once. Here we may need to pass, if necessary, to a subsequence and delete some of the terms in the Kegel sequence. Again passing to a subsequence, if necessary, we may assume that there exists a prime, say $n$, which does not appear as a characteristic in the list and is different from $p$. Let $x$ be an element of order $n$ so that $x$ becomes a semisimple element in all the classical simple groups $\bar{G}_{i} / Z\left(\bar{G}_{i}\right)$. Then by [6, Theorem $C$ (iv)] we get $C_{\bar{G}_{t}}(x) \in T_{n+\left[\frac{4}{n}\right]}$. Here $T_{n}$ denotes the class of locally finite groups having a series of finite length in which there are at most $n$ non-abelian simple factors and the rest are locally soluble. (For details see [6, Section 2].) But by coprime action $C_{\tilde{G}_{t}}(x)$ equals $C_{G_{l}}(x) O_{p}\left(G_{i}\right) / O_{p}\left(G_{i}\right)$. This implies by [6, Lemma 2.1] that $C_{G_{i}}(x)$ is in $T_{n+\left[\frac{4}{n}\right]}$. Then by [6, Lemma 2.3] it follows that $C_{G}(x)$ is in $T_{n+\left[\frac{4}{n}\right]}$ and involves an infinite non-linear finite locally simple group.

In any case the centralizer of one of the elements $x$ involves an infinite non-linear locally finite simple group and this is impossible by [8, Lemma 4.1]. Therefore there exists no simple LFBT-group.

Proposition 3. Let $G$ be a LFBT-group. If $H$ is almost locally p-soluble, then $G$ is almost locally p-soluble. In particular $G$ is a p-group and every proper normal subgroup is nilpotent of finite exponent.

Proof. Let $p$ be a prime. If $K$ is any locally finite group, let $K_{p}$ be the product of all normal locally $p$-soluble subgroups of $K$. Then $K_{p}$ is locally $p$-soluble and $K / K_{p}$ has no non-trivial locally $p$-soluble normal subgroup.

Suppose $H$ is almost locally $p$-soluble. If $K$ is a proper normal subgroup of $G$, then 
$\left|K: H_{p} \cap K\right|$ must be finite, so that $K / K_{p}$ is finite. By (1) $[K, G] \leq K_{p}$, and so $K$ must equal $K_{p}$. By (3) and (8), $G$ must equal $G_{p}$. i.e. $G$ is locally $p$-soluble. Now the rest of the theorem follows from [8, Theorem 1.1].

Therefore the restriction of local $p$-solubility on $G$ of $[8$, Theorem 1.1] is reduced to the restriction of almost local p-solubility of $H$.

Corollary 1. Let $G$ be a LFBT-group. If $H$ is nilpotent, then $G$ is a p-group and each proper subgroup of $G$ is nilpotent.

Proof. By Proposition 3 and (8) $G$ is a p-group and a union of nilpotent proper normal subgroups. Let $X$ be any proper subgroup of $G$. Then $|X: X \cap H|<\infty$. Let $Y$ be a normal subgroup of $X$ of finite index and contained in $X \cap H$. Then $X=F^{X} Y$ for some finite subgroup $F$ of $X$. Hence $X$ is nilpotent.

Proof of Proposition 1. Assume that $H$ is abelian. By Theorem 1, $G$ is not simple. By (8) $G$ is a union of proper normal subgroups. Let $N$ be a proper normal subgroup of $G$. Let $A$ be a normal subgroup of $N$ of finite index and contained in $H$. Let $B$ be the FC-radical of $N$. Then $B / Z(B)$ is finite, so $N / Z(B)$ is as well. $(G / Z(B)) / C_{(G / Z(B))}(N / Z(B)) \leq A u t(N / Z(B))$ which is finite. By (1) again we have $[N, G]$ abelian. So $G^{\prime}$ is a proper subgroup. Now (i) and (ii) follows from the theorem in [4].

It remains to show that $G^{\prime}$ is abelian. Let $M=F C\left(G^{\prime}\right)$. We have $\left|G^{\prime}: M\right|$ is finite. Then the commutator group $G^{\prime} / M$ is finite. This implies that $G / M$ is an $F C$-group. It follows from (1) that $G / M$ is abelian. Thus $M=G^{\prime}$. But then, $G^{\prime}$ is an abelian by finite $F C$-group. Therefore $G^{\prime}$ is central by finite. However $G^{\prime}$ does not have a subgroup $N$ of finite index. Then we get $G^{\prime}$ is abelian. Now (iii) follows from the theorem in [4].

Lemma 1. If there exists a finitary LFBT-group on a set $\Omega$, then $G=G^{\prime}$.

Proof. Assume if possible that $G$ is a finitary LFBT-group on the set $\Omega$, and $G \neq G^{\prime}$. Let $\Delta$ be an orbit of $G^{\prime}$ containing $\alpha \in \Omega$. Then $\Delta$ is a finite $G$-block. Let $\Xi=\{\Delta g: g \in G\}$ be the set of distinct orbits of $G^{\prime}$ on $\Omega$. Then $G$ acts on $\Xi$ transitively and there exists a homomorphism $\rho$, from $G$ to finitary symmetric group on $\Xi$. By (1) $K=\operatorname{Ker} \rho \neq G$. Then $G / K$ is an infinite abelian group acting on $\Xi$ faithfully and transitively. Now let $g K \in G / K$ and $\Delta g_{1} . g K \neq \Delta g_{1}$. Then $\Delta g_{1} g_{2} . g K \neq \Delta g_{1} g_{2}$ for all $g_{2}$. Since $G / K$ acts transitively on $\Xi, g K$ moves every element of $\Xi$. Hence $|\operatorname{Supp} g|>|\operatorname{Supp} \rho(g)|$ which is infinite. But this is impossible as $G$ is a finitary permutation group on $\Omega$. Hence $G=G^{\prime}$.

Proof of Theorem 2. By definition the orbits of each proper subgroup of $G$ are finite. As $G$ acts transitively on $\Omega$ by (1), $\Omega$ is a countable set. Let $K$ be a proper subgroup of $G$ and let $\left\{\Omega_{i}: i=1,2,3 \ldots\right\}$ be the set of distinct orbits of $K$. Then each $\Omega_{i}$ is a finite $K$ set and $K$ acts on $\Omega_{i}$ transitively. Hence $K$ can be embedded into 
restricted direct product of finite groups. It follows that $K$ is an FC-group. This implies that $G$ is a minimal non-FC-group. But by [9] a perfect locally finite minimal nonFC-group is a p-group.

Lemma 2. If there exists a finitary LFBT-group on a set $\Delta$, then $G$ does not have a maximal G-block. Moreover $\Delta=\cup_{i=1}^{\infty} \Delta_{i}$, where $\Delta_{i}$ are finite $G$-blocks.

Proof. By [8, Lemma 2.8] $G$ is not a primitive permutation group. Hence we have non-trivial $G$-blocks

$$
\Delta_{1}<\Delta_{2}<\ldots \quad \text { and let } \delta \in \Delta_{1}
$$

Assume if possible that $\Delta_{n}$ is a maximal $G$-block. Then we have an equivalence relation corresponding to $\Delta_{n}$. Let $\rho$ be the set of equivalence classes corresponding to the equivalence relation of $\Delta_{n}$. Then $G$ acts on $\rho$ transitively and $\Delta_{n}$ is a maximal $G$-block of the permutational pair $(G, \Delta)$ so $(G, \rho)$ is a primitive permutation group and the stabilizer of a point in $\rho$ is a maximal subgroup of $G$ but this is impossible by [8, Lemma 2.10]. Hence the existence of maximal $G$-block $\Delta_{n}$ is impossible. Therefore we have an infinite tower of $G$-blocks $\Delta_{1}<\Delta_{2}<\Delta_{3}<\ldots$ and $\cup_{i=1}^{\infty} \Delta_{i}=\Delta$.

The following lemma might have an independent interest in finitary permutation groups.

We use [3] as a reference for the properties of the wreath product.

Lemma 3. Let $G=\left\langle g_{i}: g_{i}^{p}=1, \quad i=1,2,3, \ldots\right\rangle$ be a transitive finitary permutation group on a set $\Delta$ and $\Delta=\cup_{i=1}^{\infty} \Delta_{i}$ where $\Delta_{1}<\Delta_{2} \ldots$ and $\Delta_{i}$ are finite $G$-blocks. Then $G$ has a subgroup isomorphic to $W r^{N} C_{p}$.

Proof. Let $g$ be an element of $G$ of order $p$. Then there exists a $G$-block $\Delta_{i}$ such that Supp $g \subseteq \Delta_{i_{1}}$. Since $G$ is transitive not all $g_{i}, \quad i=1,2,3 \ldots$, can stabilize $\Delta_{i_{1}}$. So there exists $g_{i_{1}}$ such that $g_{i_{1}}^{p}=1$ and $\Delta_{i_{1}} g_{i_{1}} \neq \Delta_{i_{1}}$. Now consider $G_{i_{1}}=\left\langle g, g_{i_{1}}\right\rangle$. The elements $g$ and $g^{\theta_{i_{1}}^{n}}, \quad 1 \leq n \leq p-1$ commute. Since $\left\langle g^{\theta_{i_{1}}}\right\rangle$ and $\left\langle g^{g_{i_{1}}^{m}}\right\rangle, \quad 1 \leq n, m \leq p-1, \quad n \neq m$ moves distinct points of $\Delta$, the intersection $\left\langle g^{\theta_{i_{1}}^{n}}\right\rangle \cap\left\langle g^{\theta_{i_{1}^{m}}^{m}}\right\rangle=1$ for all $n \neq m$ and

$$
\left\langle g, g^{g_{i_{1}}}, g^{g_{i_{1}}^{2}}, \ldots, g^{g_{i_{1}}^{p-1}}\right\rangle=\langle g\rangle \times\left\langle g^{g_{i_{1}}}\right\rangle \times\left\langle g^{g_{i_{1}}^{2}}\right\rangle \times \ldots \times\left\langle g^{g_{i_{1}}^{p-1}}\right\rangle
$$

and

$$
\left\langle g, g_{i_{1}}\right\rangle=\langle g\rangle \times\langle g\rangle^{g_{i_{1}}} \times\langle g\rangle^{g_{i_{1}}^{2}} \times \ldots \times\langle g\rangle^{g_{i_{1}}^{p-1}} \times\left\langle g_{i_{1}}\right\rangle
$$

Hence $\left\langle g, g_{i_{1}}\right\rangle \cong\langle g\rangle_{2}<\left\langle g_{i_{1}}\right\rangle \cong C_{p} \prec C_{p}$. As Supp $x y \subseteq$ Supp $x \cup \operatorname{Supp} y$, again by (9) there exists $\Delta_{i_{2}}$ such that Supp $G_{i_{1}} \subseteq \Delta_{i_{2}}$ and $\left|\Delta_{i_{2}}\right|<\infty$ so there exists $g_{i_{2}} \in G$ such that $g_{i_{2}}^{p}=1$ and $\Delta_{i_{2}} \cap \Delta_{i_{2}} g_{i_{2}}=\emptyset$. Then the elements of $G_{i_{1}}$ and $\left\langle g_{i_{2}}\right\rangle$ do not commute but, for 
any $x \in G_{i_{1}}$, the element $x$ and $x^{g_{i_{2}}^{n}}, \quad 1 \leq n \leq p-1$ commute. Then

$$
\begin{array}{r}
\left\langle G_{i_{1}}, g_{i_{2}}\right\rangle=G_{i_{1}} \times G_{i_{2}}^{g i_{2}} \times \ldots \times G_{i_{1}}^{\left(g_{i_{2}}\right)^{p-1}} \times\left\langle g_{i_{2}}\right\rangle \\
G_{i_{2}}=\left\langle g, g_{i_{1}} g_{i_{2}}\right\rangle \cong G_{i_{1}} 2\left\langle g_{i_{2}}\right\rangle
\end{array}
$$

We can continue this process since $G_{i j}$ is a finite group and we have a tower of finite $G$-blocks. Then we have

$$
G_{i_{j}} \cong G_{i_{j-1}}>\left\langle g_{i_{j}}\right\rangle \text { and } G_{i_{1}}<G_{i_{2}}<\ldots
$$

In order to simplify the notation let us suppress the $i$ in the subscripts i.e. we have $G_{i} \cong W_{i}$ where $W_{i}=C_{p} 2 C_{p} 2 \ldots 2 C_{p}$ ( $i$ times). Suppose we have an isomorphism $\psi_{j}: G_{j} \rightarrow W_{j}$. We need to extend this isomorphism $\psi_{j}$ to an isomorphism $\psi_{j+1}$ between $G_{j+1}$ and $W_{j+1}$. Let

$$
\psi_{j+1}: \prod_{t=0}^{p-1} x_{t}^{\left(g_{j+1}^{t}\right)} g_{j+1}^{s} \rightarrow \prod_{t=0}^{p-1} \psi_{j}\left(x_{t}\right)^{w_{j+1}^{t}} w_{j+1}^{s}, \quad x_{t} \in G_{j}, \quad(0 \leq s \leq p-1)
$$

Clearly $\psi_{j+1}$ is a well defined map from $G_{j+1}$ to $W_{j+1}$. It follows that $\psi_{j+1}$ is an isomorphism.

Then $\left\{G_{j}, \psi_{j}: j=1,2,3, \ldots\right\}$ is a direct system and $\left.\psi_{j+1}\right|_{G_{j}}=\psi_{j}$. Let $\Psi:\left(K=: \cup G_{i}\right) \rightarrow W$. If $g \in K$ there exists $i$ such that $g \in G_{i}$, then $\psi(g)=\psi_{i}(g)$. $\Psi$ is an isomorphism and $K \leq G$.

Let $\Omega_{i}=\left\{\Delta_{i}, \Delta_{i} g_{i}, \ldots, \Delta_{i} g_{i}^{p-1}\right\}$. Then $\left\langle g_{i}\right\rangle$ acts on $\Omega_{i}$ transitively. Let

$$
\Omega=D r_{i \in N} \Omega_{i}
$$

Now as in [3]; choose $\left(\Delta_{i}\right)_{i \in N}$ as the reference point. Then every $g_{i}^{n}, \quad i=1,2,3, \ldots$, $1 \leq n \leq p$ gives a permutation of $\Omega$ so

$$
\left\langle g_{i} \theta_{i}: g_{i}^{p}=1, i \in N\right\rangle
$$

acts on $\Omega$ as in the definition and hence

$$
\left\langle g_{i} \theta_{i}: g_{i}^{p}=1, i \in N\right\rangle=W r^{N} C_{p}
$$

where $\theta_{i}:\left\langle g_{i}\right\rangle \rightarrow \operatorname{Sym}(\Omega)$. Hence $K$ is the required subgroup of $G$.

Proof of Theorem 3. Assume to the contrary that $G$ is a LFBT-group. By Lemma 2 we have 


$$
\Delta_{1}<\Delta_{2}<\Delta_{3}<\ldots \text { and } \bigcup_{i=1}^{\infty} \Delta_{i}=\Delta \text {. }
$$

By Lemma $3 G$ has a subgroup $K$ isomorphic to $W r^{N} C_{p}$. If $K$ is a proper subgroup of $G$, then bare transitivity of $G$ implies that $K$ is a residually finite group and hence $K^{\prime}$ is residually finite. But by $[3$, p. 173$] K^{\prime}$ is a perfect $p$-group hence this is impossible. If $K=G$, then $G$ has proper subgroups isomorphic to $K$ but this is impossible by the above paragraph. Hence the assumption that $G$ is a LFBT-group lead us a contradiction.

\section{REFERENCES}

1. V. V. BeLyaEv, Locally finite simple groups as a product of two inert subgroups, Algebra i Logika 31 (1992), 360-368 (Russian); translated in Algebra and Logic 31 (1992), 216-221.

2. V. V. BeLYAEV, Semisimple periodic groups of finitary transformations. Algebra $i$ Logika 32 (1992), 17-33 (Russian); translated in Algebra and Logic 32 (1993), 8-16.

3. P. Hall, Wreath Products and Characteristically Simple Groups, Math. Proc. Cambridge Philos. Soc. 58 (1962), 170-184.

4. B. HaRTLEY, On the normalizer condition and barely transitive permutation groups, Algebra i Logika 13 (1974), 589-602 (Russian); translated in Algebra and Logic 13 (1974), 334-340.

5. B. Hartley, A note on normalizer condition, Math. Proc. Cambridge Philos. Soc. 74 (1973), 11-15.

6. B. HartLey and M. KuzucuoĞLu, Centralizers of elements in locally finite simple groups, Proc. London Math. Soc. (3) 62 (1991), 301-324.

7. M. KuzucuoŏLu, Centralizers of semisimple subgroups in locally finite simple groups, Rend. Sem. Mat. Univ. Padova, 92 (1994), 79-90.

8. M. KuzucuoĞLu, Barely Transitive Permutation Groups, Arch. Math. 55 (1990), $521-532$.

9. M. KuzucuoĞLu and R. Phillıps, Locally finite minimal non-FC-groups, Math. Proc. Cambridge Philos. Soc. 105 (1989), 417-420.

Department of Mathematics

Middle EAST TECHNICAL UNIVERSITY

06531, ANKARA

TURKEY

E-mail: matmah@rorqual.cc.metu.edu.tr 